\title{
Monte Carlo Investigation of Diffusion of Receptors and Ligands that Bind Across Opposing Surfaces
}

\author{
Philippos K. Tsourkas and Subhadip Raychaudhuri \\ Department of Biomedical Engineering, University of California, Davis, One Shields Avenue, Davis, CA 95616, USA
}

(Received 27 April 2010; accepted 5 August 2010; published online 2 September 2010)

Associate Editor Scott I. Simon oversaw the review of this article.

\begin{abstract}
Studies of receptor diffusion on a cell surface show a variety of behaviors, such as diffusive, sub-diffusive, or super-diffusive motion. However, most studies to date focus on receptor molecules diffusing on a single cell surface. We have previously studied receptor diffusion to probe the molecular mechanism of receptor clustering at the cell-cell junction between two opposing cell surfaces. Here, we characterize the diffusion of receptors and ligands that bind to each other across two opposing cell surfaces, as in cell-cell and cell-bilayer interactions. We use a Monte Carlo method, where receptors and ligands are simulated as independent agents that bind and diffuse probabilistically. We vary receptor-ligand binding affinity and plot the moleculeaveraged mean square displacement (MSD) of ligand molecules as a function of time. Our results show that MSD plots are qualitatively different for flat and curved interfaces, as well as between the cases of presence and absence of directed transport of receptor-ligand complexes toward a specific location on the interface. Receptor-ligand binding across two opposing surfaces leads to transient sub-diffusive motion at early times provided the interface is flat. This effect is entirely absent if the interface is curved, however, in this instance we observe sub-diffusive motion. In addition, a decrease in the equilibrium value of the MSD occurs as affinity increases, something which is absent for a flat interface. In the presence of directed transport of receptorligand complexes, we observe super-diffusive motion at early times for a flat interface. Super-diffusive motion is absent for a curved interface, however, in this case we observe a transient decrease in MSD with time prior to equilibration for high-affinity values.
\end{abstract}

Keywords-Sub-diffusion, Anomalous diffusion, Single particle tracking, Cell membrane, Cell-cell interaction, Receptor clustering, Computational modeling, Directed transport, Mean square displacement, Affinity.

Address correspondence to Subhadip Raychaudhuri, Department of Biomedical Engineering, University of California, Davis, One Shields Avenue, Davis, CA 95616, USA. Electronic mail: raychaudhuri@ucdavis.edu

\section{INTRODUCTION}

The lateral diffusion of receptor molecules on a cell membrane is of great importance to many cellular processes. ${ }^{6,23}$ Diffusion is critical to processes such as receptor segregation, ${ }^{16}$ the formation of supramolecular signaling complexes, ${ }^{15,39-42}$ receptor internalization, ${ }^{23}$ and association of receptors with lipid rafts, ${ }^{20,22}$ among others. Diffusion of receptors on cellular membranes has been extensively studied using experimental techniques such as fluorescence recovery after photobleaching (FRAP) and single-particle tracking (SPT), ${ }^{20,35}$ and theoretical techniques such as Monte Carlo simulation. . $26,27,29-32,37,42,44$ Such experimental and theoretical studies demonstrated that the cell membrane is a highly complex and heterogeneous environment. It is now well-known that cell surfaces are divided into compartments, such that it is considerably more difficult for surface molecules to diffuse between compartments than within a compartment (hop diffusion). ${ }^{21}$ Cell membranes also contain inert obstacles and reactive immobile membrane proteins (traps), as well as sphingolipid-enriched regions (rafts) that can hinder the diffusion of mobile membrane molecules. Experimental FRAP studies have shown that diffusion on the cell membrane is one to two orders of magnitude slower than on artificial lipid bilayers. ${ }^{35}$ In addition, experimental SPT and theoretical studies have shown that diffusion on a surface with obstacles, such as a cell membrane, can deviate from pure (or classical) diffusion and is in many cases sub-diffusive, $, 20,26,31-35,42,43$ i.e., the mean square displacement (MSD) varies with time in sub-linear fashion so that $\mathrm{MSD}=D \cdot t^{\alpha}$, where $\alpha<1$ (also known as anomalous diffusion).

Most receptor-mediated cellular processes of interest also involve some form of chemical reaction, whether it is receptor oligomerization, binding to reactive membrane proteins on the same surface, or binding to 
soluble or membrane-bound ligands on another cell surface. Several studies of diffusion on a cell membrane include reaction, however, most of these studies deal exclusively with reactions that occur on the same surface on which the receptors are diffusing, such as receptor oligomerization, or binding of receptors to traps. $^{7,11,17,31,33,34,37,44}$ Yet, understanding how receptor-ligand binding on opposing surfaces impacts diffusion is of great interest to the study of cell-cell communication processes, as happens, for example, in the immune system during antigen recognition. In this instance, the lymphocyte receptor (in the case of $\mathrm{T}$ cells it is the T cell receptor, or TCR, in the case of B cells it is the $\mathrm{B}$ cell receptor, or BCR) binds to antigen arrayed on the surface of an antigen presenting cell (APC) and becomes activated depending on the affinity with which it binds the antigen. Receptor-antigen complexes cluster at the center of the cell interface, surrounded by integrin-integrin complexes, forming the supramolecular activation complex known as the "immunological synapse," a key step in the lymphocyte activation process. ${ }^{1,5,14,19,25,46}$ Diffusion of receptors and ligands (in this case, antigens and integrins) plays a crucial role in the formation of the immunological synapse. ${ }^{15,40-42}$ During the initial phase immunological synapse formation, receptors form nanoscale micro-clusters on lipid raft domains, whose formation is also crucially modulated by diffusion of receptors and ligands. ${ }^{10,13,36,38}$

In previous work, we used a Monte Carlo simulation method to show that the MSD of receptors and ligands that bind across opposing surfaces can be used as a tool to estimate the order of magnitude of the diffusion coefficient of receptor-ligand complexes, something that is difficult to measure experimentally. ${ }^{42}$ In that work, we showed that the MSD increased in a sub-diffusive manner with time and that sub-diffusive motion is highly dependent on the affinity of receptorantigen binding. However, we did not thoroughly investigate the causes of affinity-dependent sub-diffusive motion, nor did we attempt characterize the motion or study its equilibrium behavior. In a separate study, we have shown that directed transport of receptors can be a mechanism for immunological synapse formation in B cells. ${ }^{40}$ However, in that work, the directed transport of receptors was not probed quantitatively through MSD plots.

In this work, we quantitatively investigate the motion of receptors that bind to diffusing monovalent ligands on an opposing surface. Though this may seem equivalent to binding of receptors to traps on the same surface, an additional complication in this case is that the geometry of the cell-cell interface may vary. The shape of the interface, in turn, may strongly affect the motion of receptors that bind ligands on an opposing surface. For example, it has been shown that thermal roughness in membranes can reduce the diffusion coefficient of membrane-bound molecules by a factor of $2 .{ }^{18}$ Thus, in contrast to previous work, we study both cell-cell interfaces where the cell surfaces exhibit strong curvature, as is the case during the early stages of a cell-cell interaction (e.g., through protrusions ${ }^{10,13,36,38}$, and as a completely flat surfaces, as is the case during the late stages of cell-cell interaction. ${ }^{10,13,36,38}$ Three potential sources of deviation from classical diffusion (MSD $=D \cdot t$ ) were investigated: receptor-ligand binding, interface curvature, and cytoskeletally driven transport of receptors to the center of the contact zone, as observed in a number of experiments. $^{7,9,10,13,16,29,36,38,45}$ In order to gage the effect of each of these components on diffusion, controlled virtual experiments were performed in which each of these system properties were varied individually.

We show that for a flat contact interface, receptorligand binding causes transient sub-diffusive behavior at early times that disappears once kinetic equilibrium of receptor-ligand binding is reached. For a curved interface, this effect is absent, but we show that surface curvature alone can induce sub-diffusive behavior at late times, so that higher affinity values converge to a lower equilibrium MSD value. By contrast, for a flat interface, the MSD converges to the same equilibrium value, regardless of receptor-ligand affinity or other parameter values. Directed transport causes superdiffusive motion (MSD $=D \cdot t^{\alpha}$, where $\alpha>1$ ) at early times for a flat interface, but not for a curved interface. At late times, directed transport of receptors induces sub-diffusive motion in an affinity-dependent manner irrespective of the geometry of the cell-cell interface, in a manner that is qualitatively similar to undirected transport on a curved interface.

\section{METHOD}

Our method is a Monte Carlo simulation procedure similar to our previous work..$^{28,41,42}$ The two opposing cell surfaces are simulated as discrete lattices, on which receptors and ligands randomly diffuse and react with each other according to probabilistic parameters. A distinguishing feature of our method is a mapping between the probabilistic parameters of the Monte Carlo simulation and their physical counterparts, which enables us to physically interpret our results. ${ }^{41}$

\section{Setup}

Cell surfaces are simulated as discrete, $N \times N$ Cartesian lattices. No two molecules may occupy the 
same node, hence we choose a spacing between nodes of $10 \mathrm{~nm}$, about equal to the exclusion radius of lymphocyte receptor molecules. Each node has four neighbors. In this work, we simulate diffusion within a single $3 \times 3 \mu \mathrm{m}$ membrane compartment, thus the number of nodes on one side is $N=300$. The vertical separation distance between the two surfaces, denoted by $z$, may be constant (flat interface), or it may vary with position on the lattice (curved interface). For a flat interface, we use a value of $40 \mathrm{~nm}$ (the optimum binding distance between lymphocyte receptors and antigens ${ }^{4}$ ) for the vertical separation distance, while for a curved interface we use the relation for spherical curvature:

$$
\begin{aligned}
z(x, y)= & z_{0}+R_{\text {cell }} \\
& -\left(R_{\text {cell }}^{2}-\left(\left(x-x_{0}\right)^{2}+\left(y-y_{0}\right)^{2}\right)\right)^{1 / 2}
\end{aligned}
$$

Here $R_{\text {cell }}$ denotes the cell radius, which is set to $6 \mu \mathrm{m}$, a typical radius for a lymphocyte, while $z_{0}$ is set to $40 \mathrm{~nm}$. At the center of the contact interface, the vertical separation distance is thus equal to $40 \mathrm{~nm}$, but increases according to Eq. (1) away from the center. ${ }^{41}$ For a $6 \mu \mathrm{m}$ cell radius, the vertical separation distance at the corners of a $3 \times 3 \mu \mathrm{m}$ area is $\sim 400 \mathrm{~nm}$, which is an order of magnitude greater than the optimum binding distance between receptors and ligands. The choice of a $3 \times 3 \mu \mathrm{m}$ area is thus large enough to include the entire region where receptor-ligand binding is possible on a curved interface, and is also large enough to assume a zero net flux at the domain boundaries, which we simulate using reflective boundary conditions. ${ }^{41,42}$ The use of reflective boundary conditions is also analogous to diffusion within a single membrane compartment with low probability of escape, and allows us to study equilibrium behavior. To simulate membrane protrusions, we used a smaller radius of curvature, though this did not qualitatively alter our results. The molecules on surface 1 are denoted as "receptors," while those of surface 2 are denoted as "ligands." The spatial parameters of our model are summarized in Table 1.

\section{Random Sampling}

At the start of a simulation run, $R_{0}=1000$ receptors and $L_{0}=1000$ ligands are uniformly distributed over their respective surfaces (the number is obtained by dividing the typical value of $10^{4}-10^{5}$ receptors/cell by the surface area of a cell with radius $6 \mu \mathrm{m}$ and multiplying the result by $3 \times 3 \mu \mathrm{m})$. At each time step, molecules in the population are individually sampled in a random manner to undergo either diffusion or reaction, determined by means of a coin toss with
TABLE 1. Spatial dimensions of the model.

\begin{tabular}{ll}
\hline Parameter & \multicolumn{1}{c}{ Value } \\
\hline Size of contact region & $3 \times 3 \mu \mathrm{m}$ \\
Number of nodes $(M)$ & $300 \times 300$ \\
Nodal spacing & $10 \mathrm{~nm}$ \\
Cell radius $\left(R_{\text {cell }}\right)$ & $6 \mu \mathrm{m}$ \\
Minimum vertical separation distance $\left(z_{0}\right)$ & $40 \mathrm{~nm}$ \\
\hline
\end{tabular}

probability 0.5 . A number $S$ of diffusion/reaction trials is performed during every time step. The number of trials $S$ is set equal to the total number of molecules (free and complexes) at the beginning of each time step, and the simulation is run for a number of time steps $T$. Each Monte Carlo time step corresponds to $10^{-3} \mathrm{~s}$, as shown in Tsourkas et al. ${ }^{41}$

\section{Diffusion}

If a molecule has been selected to undergo diffusion, a random number trial with probability $p_{\text {diff }}$ is used to determine whether the diffusion move will occur. The diffusion probability $p_{\text {diff }}$ is directly analogous to the diffusion coefficient $D .^{41}$ The probability of diffusion of free molecules (receptors and ligand) is denoted by

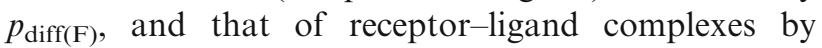
$p_{\text {diff(C) }}$. If the trial with probability $p_{\text {diff }}$ is successful, one of the four neighboring nodes is selected at random and checked for occupancy. The diffusion move is only carried out if the target node is vacant. For receptor-ligand complexes, the target nodes on both surfaces are checked. The diffusion coefficient of receptor molecules on a cell membrane has been experimentally measured to be of the order of $0.1 \mu \mathrm{m}^{2} / \mathrm{s} .^{12,24}$ The probability of diffusion of receptor-ligand complexes has not been measured experimentally, but receptor-ligand complexes are generally believed to diffuse an order of magnitude slower than free molecules. $^{24,38}$ Since free receptor and ligand molecules are the fastest diffusing species, we set $p_{\text {diff(F) }}=1$, and $p_{\text {diff(C) }}=0.1$.

Because of the intricacies involved in explicitly modeling the cytoskeleton, cytoskeletally mediated transport of receptor-ligand complexes toward the center of the contact zone is simulated in an indirect manner by biasing the diffusion of receptor-ligand complexes toward the center. We define a biasing factor $\eta$ that we multiply $p_{\text {diff(C) }}$ by if the target node is closer to the center of the domain than the molecule's current location. The case $\eta=1$ corresponds to purely random motion, with biased motion toward the center increasing with larger $\eta$. The use of a biasing factor does not account for signaling-driven effects, such as stochastic variations in the time scale of the onset of 
directed diffusion. However, simulating cytoskeletally mediated transport in this manner is equivalent to explicitly simulating attachment, detachment, and transport by the cytoskeleton, as shown in our previous work. ${ }^{40}$

\section{Reaction}

If selected for a reaction, a receptor (or ligand) may bind to a ligand (or receptor) with probability $p_{\text {on }}$ and form a receptor-ligand complex, provided a ligand (or receptor) happens to be located on the facing node on the opposing surface. If a receptor-ligand complex is selected for a reaction, it may dissociate with probability $p_{\text {off }}$. The probabilities $p_{\text {on }}$ and $p_{\text {off }}$ are directly analogous to the kinetic constants $k_{\text {on }}$ and $k_{\text {off }}$, and their ratio, denoted as $P_{\mathrm{A}}$, is directly analogous to the association constant $K_{\mathrm{A}}$. A detailed description of the mapping of $p_{\text {on }}, p_{\text {off }}$, and $P_{\mathrm{A}}$ to their respective physical counterparts, $k_{\text {on }}, k_{\text {off }}$, and $K_{\mathrm{A}}$ can be found in our previous work. ${ }^{41}$

For a flat interface, $p_{\text {on }}$ and $p_{\text {off }}$ are constants, but for a curved interface, $p_{\text {on }}$ and $p_{\text {off }}$ vary as the square of the difference between the local vertical separation distance and the optimum receptor-ligand bond length $\left(z-z_{0}\right)^{2}$ (hence a fourth-order dependence on $x$ and $y$ ), in accordance with the well-known linear spring model. ${ }^{2,4,8,41,42}$ The probability of binding $p_{\text {on }}$ is given by:

$$
p_{\text {on }}(z)=p_{\text {on }}^{\max } \exp \left(-\frac{\kappa\left(z-z_{0}\right)^{2}}{2 k_{\mathrm{B}} T}\right)
$$

The bond is modeled as a mechanical spring with stiffness $\kappa$ and equilibrium length $z_{0}$, while $k_{\mathrm{B}}$ denotes the Boltzmann constant $\left(1.34 \times 10^{-23} \mathrm{~J} / \mathrm{K}\right)$ and $T$ is the temperature $(\sim 300 \mathrm{~K})$. Similarly, the dissociation probability of a receptor-ligand complex, $p_{\text {off (i) }}$, is given by:

$$
p_{\text {off }}(z)=p_{\text {off }}^{\min } \exp \left(\frac{\kappa\left(z-z_{0}\right)^{2}}{2 k_{\mathrm{B}} T}\right)
$$

Since $p_{\text {on }}$ and $p_{\text {off }}$ are analogous to $k_{\text {on }}$ and $k_{\text {off }}$, we can obtain the probabilistic analog to the association constant $K_{\mathrm{A}}$, denoted as $P_{\mathrm{A}}$, by dividing Eq. (2) by Eq. (3):

$$
\begin{aligned}
P_{\mathrm{A}}(z) & =\frac{p_{\mathrm{on}}^{\max }}{p_{\mathrm{off}}^{\min }} \exp \left(-\frac{\kappa\left(z-z_{0}\right)^{2}}{2 k_{\mathrm{B}} T}\right) \\
& =P_{\mathrm{A}}^{\max } \exp \left(-\frac{\kappa\left(z-z_{0}\right)^{2}}{2 k_{\mathrm{B}} T}\right)
\end{aligned}
$$

Thus, for a curved interface, $p_{\text {on }}$ reaches its maximum value at the center of the contact zone, decreasing as one moves away from the center, while $p_{\text {off }}$ is a minimum at the center and increases away from it, reaching a value of 1 well before the domain boundary is reached. ${ }^{41}$ Thus, in our model receptorligand complexes are effectively restricted to a region within a certain distance from the center of the domain. The boundary of this area is probabilistic and emerges naturally from the kinetic parameters and interface geometry rather than being arbitrarily set. Setting a strict boundary past which receptor-ligand complexes could not diffuse would introduce an element of arbitrariness that is absent from our current binding model. If the boundary were set too close to the center, this would lead to an artificially high concentration of receptor-ligand complexes near the center. If the boundary were set far from center, to the outermost limit of the region where binding is possible in our current model, there would be no difference in results between the two binding models. Using a Metropolis scheme, as in Krobath et al., ${ }^{18}$ where the probability of diffusion is proportional to the change in energy associated with the proposed diffusion hop, would result in behavior similar to our model, however, at a greater computational cost.

\section{Affinity Variation}

Simulations are performed for flat and curved cellcell interface, and biased and unbiased diffusion, resulting in four overall sets of experiments. Five values of receptor-ligand binding affinity are used in the simulations for unbiased diffusion, while three affinity and three bias values are used in the simulations for biased diffusion. As in the biological experiments our work is based on, ${ }^{5,13}$ affinity is varied by keeping $p_{\text {on }}$ constant and varying $p_{\text {off }}$. A value of $P_{\mathrm{A}}=1$ thus corresponds to $p_{\text {on }} / p_{\text {off }}=1 / 1, P_{\mathrm{A}}=10$ to $p_{\text {on }} / p_{\text {off }}=1 / 0.1$, and so forth. Also included in every simulation is a control with no receptor-ligand binding, i.e., $p_{\text {on }}=0$ and $P_{\mathrm{A}}=0$. For a curved interface, the zone where binding is possible is a fraction of the entire domain, since the vertical separation distance at the edges is too great for receptor-ligand binding to occur. For this reason, it is necessary to vary affinity differently for a flat and curved interfaces. For a flat interface, the affinity range used is $P_{\mathrm{A}}=1,10,100,1000$ (corresponding to a range of $K_{\mathrm{A}}=10^{4}-10^{7} \mathrm{M}^{-1}$ ). At $P_{\mathrm{A}}=1000$, most ligands are bound to a receptor, and there is little difference between $P_{\mathrm{A}}=1000$ and $P_{\mathrm{A}}=10^{4}$. By contrast, for a curved interface, because the zone where binding is possible is a fraction of the entire domain, the affinity range used is $P_{\mathrm{A}}=1,10^{2}, 10^{4}, 10^{6}$ (corresponding to a range of $K_{\mathrm{A}}=10^{4}-10^{10} \mathrm{M}^{-1}$, the physiological affinity range in lymphocytes). 
TABLE 2. Experimentally measured parameter values and their probabilistic counterparts.

\begin{tabular}{llll}
\hline Parameter & \multicolumn{1}{c}{ Physical value } & Simulation parameter & \multicolumn{1}{c}{ Mapped value } \\
\hline$K_{\mathrm{A}}$ (flat) & $0,10^{4}-10^{7} \mathrm{M}^{-1}$ & $P_{\mathrm{A}}^{\max }$ & $0,1-1000$ \\
$k_{\text {on }}$ (flat) & $0,10^{7} \mathrm{M}^{-1} \mathrm{~s}^{-1}$ & $p_{\text {on }}^{\max }(\mathrm{BA})$ & 0,1 \\
$k_{\text {off }}$ (flat) & $10^{3}-1 \mathrm{~s}^{-1}$ & $p_{\text {off }}^{\min }(\mathrm{BA})$ & $1-10^{-3}$ \\
$K_{\mathrm{A}}$ (curved) & $0,10^{4}, 10^{6}, 10^{8}, 10^{10} \mathrm{M}^{-1}$ & $P_{\mathrm{A}}^{\max }$ & $0,1,10^{2}, 10^{4}, 10^{6}$ \\
$k_{\text {on }}$ (curved) & $0,10^{7} \mathrm{M}^{-1} \mathrm{~s}^{-1}$ & $p_{\text {on }}^{\max }$ & 0,1 \\
$k_{\text {off }}$ (curved) & $10^{3}, 10^{1}, 10^{-1}, 10^{-3} \mathrm{~s}^{-1}$ & $p_{\text {off }}^{\min }$ & $1,10^{-2}, 10^{-4}, 10^{-6}$ \\
Receptors & $\sim 10^{4}-10^{5} / \mathrm{cell}^{2}$ & $R_{0}$ & 1000 molecules \\
Ligands & $\sim 10^{4}-10^{5} / \mathrm{cell}^{2}$ & $L_{0}$ & 1000 molecules \\
$D_{\text {free molecules }}$ & $\sim 0.1 \mu \mathrm{m}^{2} / \mathrm{s}^{12,24}$ & $p_{\text {diff(F) }}$ & 1.0 \\
$D_{\text {complexes }}$ & $\sim 0.01 \mu \mathrm{m}^{2} / \mathrm{s}^{24,38}$ & $p_{\text {diff(C) }}$ & 0.1 \\
$\kappa$ & $40 \mu \mathrm{N} / \mathrm{m}^{4}$ & $\kappa$ & Same as meas. value \\
\hline
\end{tabular}

The molecular parameters used in our model are listed in Table 2. Parameter values found in the literature are given on the left side of Table 2, while the appropriately mapped forms used in our simulation, where applicable, are listed on the right side of Table 2.

\section{RESULTS}

To characterize diffusion, we track the square displacement of ligand molecules over time, defined as the square of the Euclidean distance between a ligand's current location and its location at the start of the simulation. As trajectories and the square displacement can vary widely from molecule to molecule (many orders of magnitude), we collect the square displacement for all 1000 ligand molecules in one simulation run and plot the molecule-averaged, or mean square displacement (MSD). We also tabulate the molecule-averaged distance from the center of the contact zone of ligand molecules as a measure of clustering.

\section{Unbiased Diffusion}

\section{Flat Contact Interface}

The plot of MSD as a function of time for unbiased diffusion and a flat contact interface is shown in Fig. 1. Note the different time and MSD scales in each of the figure panels. The evolution of MSD up to $t=10^{4}$ time steps is shown in Fig. 1a, up to $t=10^{5}$ time steps in Fig. 1b, up to $t=10^{6}$ time steps in Fig. 1c, and up to $t=10^{7}$ time steps in Fig. 1d. Before $t=10^{4}$ time steps (Fig. 1a), MSD appears to be linear with time, though the diffusion coefficient (the slope of the MSD curves) decreases with affinity. After $t=10^{4}$ time steps, sub-diffusive behavior is observed, and the MSD eventually reaches an equilibrium value set by the size of the domain. Since similar sub-diffusive behavior is observed for all affinity values, even the case of no receptor-ligand binding $\left(P_{\mathrm{A}}=0\right)$, the finite size of the domain is the sole source of the sub-diffusive behavior.

The reason for the slower increase in MSD with increasing affinity is that as affinity increases, a greater fraction of ligands are bound to receptors and diffuse with $p_{\text {diff(C) }}=0.1$ instead of $p_{\operatorname{diff}(\mathrm{F})}=1$. MSD thus increases more slowly on average for higher affinity values, the effects of finite-domain size take longer to be felt, and equilibrium takes increasingly longer to reach. For zero, low, and intermediate affinity $\left(P_{\mathrm{A}}=0,1,10\right)$, the transition to sub-diffusive motion, and equilibrium, is reached an order of magnitude sooner compared to high affinity $\left(P_{\mathrm{A}}=100,1000\right)$, as can be seen in Fig. 1b, c. Though it takes longer to reach equilibrium as affinity increases, the equilibrium MSD value itself is the same for all affinity values (Fig. 1d).

If we look at very early times in Fig. 1a, however, we discern that for the high-affinity cases, there is a very brief period during which sub-diffusive behavior is observed. This becomes immediately apparent when we plot MSD after $t=10^{2}$ and $t=10^{3}$ time steps, in Fig. 2a, b, respectively. Sub-diffusive motion is seen at early times $(t<1000$ time steps) for high-affinity binding, which transitions to linear diffusion later on. This transient sub-diffusive behavior is due to the transition from the $p_{\text {diff( } \mathrm{F})}=1$ to the $p_{\operatorname{diff}(\mathrm{C})}=0.1$ regime as ligand molecules bind receptors. Initially, all ligands diffuse with $p_{\text {diff(F) }}=1$. However, as time goes by, ligands that bind a receptor will diffuse with $p_{\text {diff(C) }}=0.1$. While the number of receptor-ligand complexes increases (i.e., before kinetic equilibrium), more and more ligands will diffuse with $p_{\text {diff(C) }}=0.1$ as time goes by, resulting in an increasingly slower increase in MSD, thereby producing sub-diffusive behavior. Once the number of receptor-ligand complexes reaches its equilibrium value, after $t \sim 200$ time steps for high affinity as shown in Fig. 3a, the transition to the $p_{\text {diff(C) }}=0.1$ regime is complete and diffusion for high-affinity binding is once again linear, albeit with a lower diffusion coefficient. 


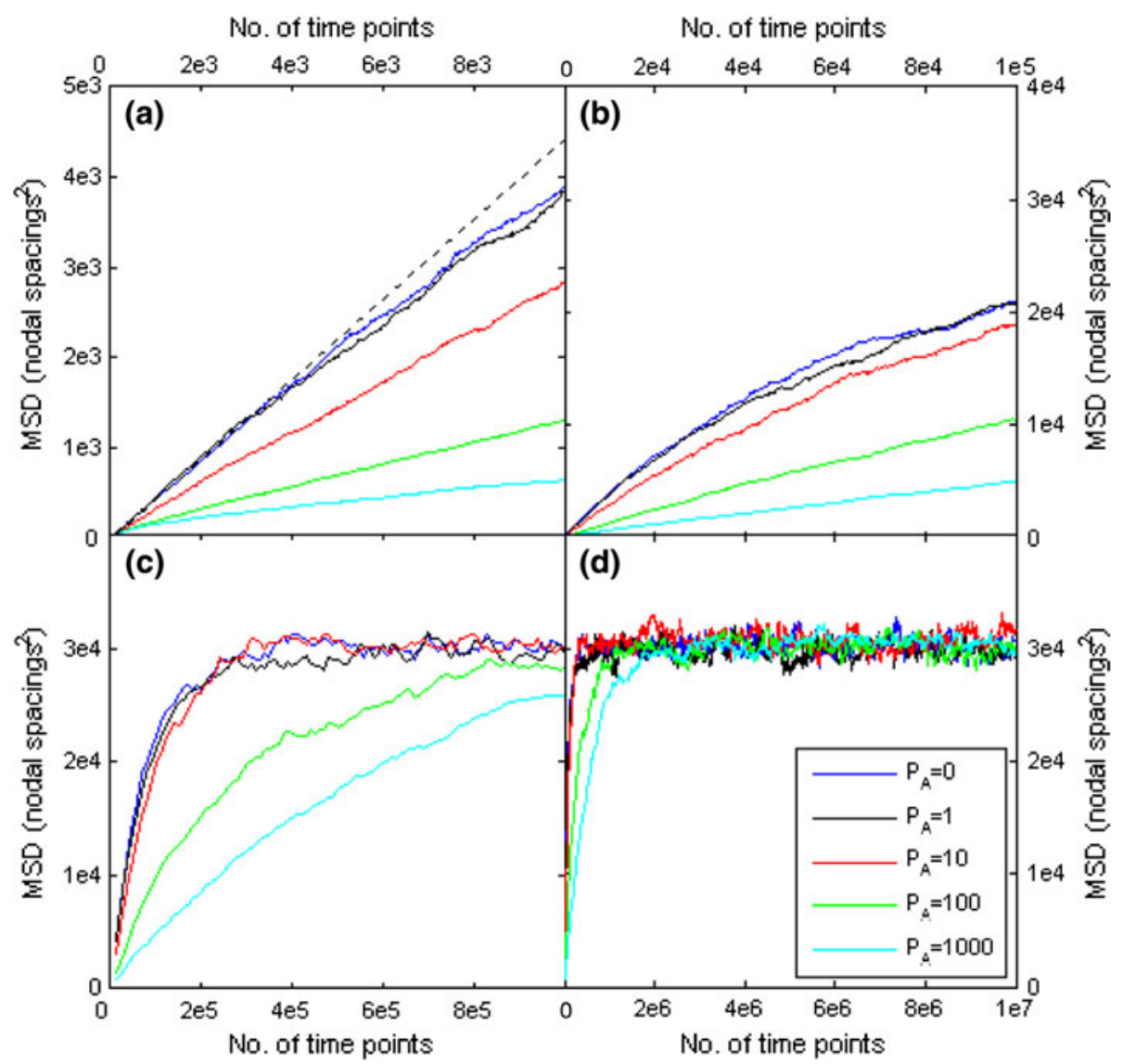

FIGURE 1. MSD as a function of time for unbiased diffusion and a flat interface. The simulation is run for $T=10^{7}$ time steps. Snapshots are taken after $t=10^{4}$ time steps (a), $t=10^{5}$ time steps (b), $t=10^{6}$ time steps (c), and $t=10^{7}$ time steps (d). Affinity takes on values of $P_{\mathrm{A}}=1,10,100,1000$, and also includes a control with $P_{\mathrm{A}}=0$. The remaining parameters are given in Tables 1 and 2. Each curve represents the MSD averaged over 1000 ligand molecules for a single simulation run. The dashed line represents classical diffusion.

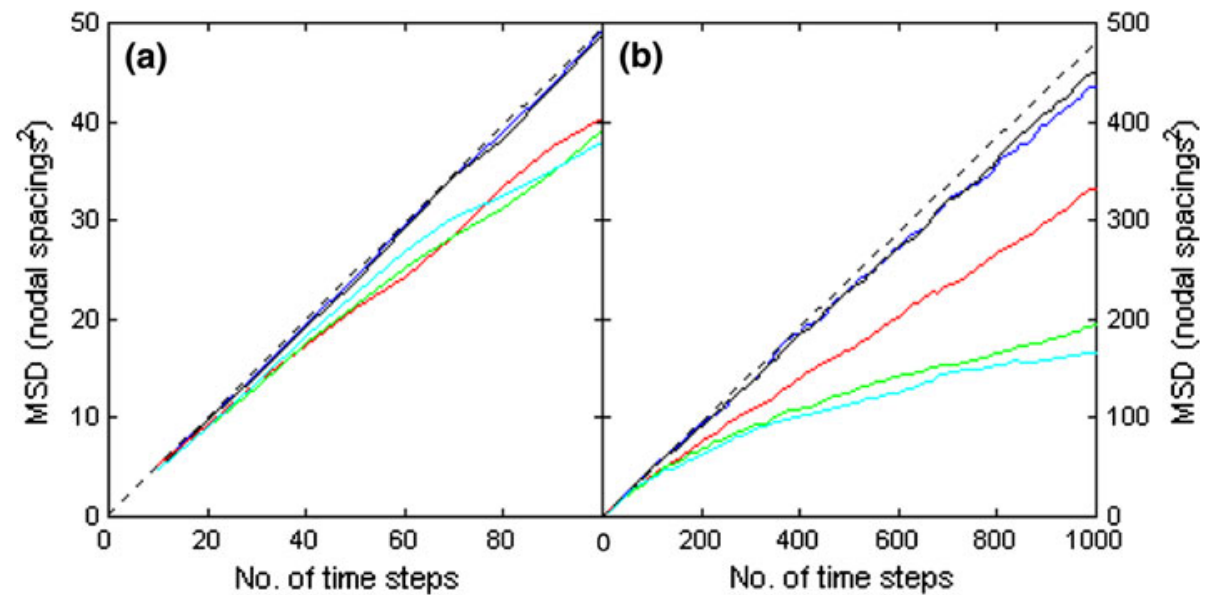

FIGURE 2. MSD as a function of time at early times for unbiased diffusion and a flat interface. The parameter values are the same as in Fig. 1, however, (a) is taken after $t=10^{2}$ time steps and (b) after $t=10^{3}$ time steps.

We thus see that receptor-ligand binding can by itself induce transient sub-diffusive behavior provided receptor-ligand complexes diffuse slower than free molecules. This effect disappears once kinetic equilibrium is reached. These results are consistent with those of Saxton for diffusion on a single surface with 


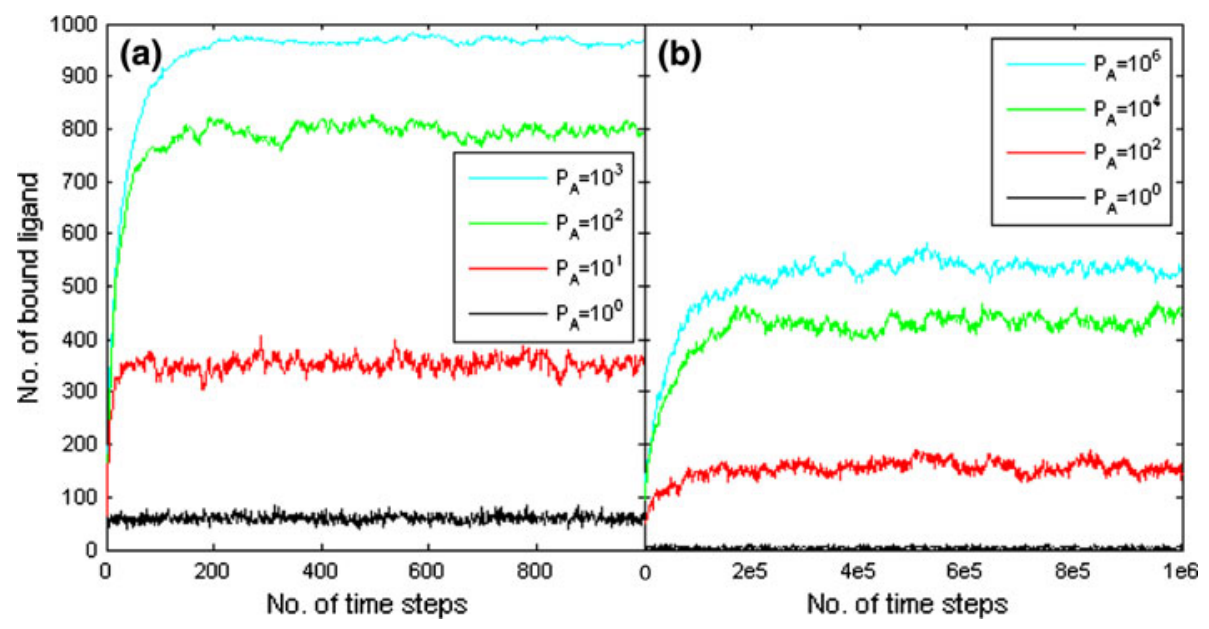

FIGURE 3. Bound ligand (i.e., no. of receptor-ligand complexes) as a function of time for a flat interface (a) and a curved interface (b), with no bias in diffusion in either case. Note the different time scale for flat and curved interfaces. For a flat interface, because equilibrium in the number of receptor-ligand complexes is reached rapidly (before $t=1000$ time steps), the plot only extends to $t=1000$ time steps rather than the full $T=10^{7}$ time steps of the simulation.

non-inert obstacles. ${ }^{31}$ This effect is entirely absent if we set $p_{\text {diff(C) }}=1$, in which case it also is not possible to distinguish between affinity values.

\section{Curved Contact Interface}

The plots of MSD as a function of time for unbiased diffusion on a curved contact interface are shown in Fig. 4. Several significant differences from the case of a flat contact interface are noted. First, the time to MSD equilibration is roughly the same for all affinity values (Fig. 4d). Second, the equilibrium MSD value for high affinity is reached roughly an order of magnitude faster than for the flat contact interface, and MSD for high affinity generally increases much faster compared to a flat contact interface. For example, at $t=10^{4}$ time steps, the MSD for high affinity for the curved interface is $\sim 3000$ (Fig. 4b), as compared to $\sim 1000$ for the flat interface (Fig. 1a). At $t=10^{5}$ time steps, the MSD for high affinity for the curved interface is significantly higher than that for the flat interface (compare Fig. 4c to Fig. 1b). By $t=4 \times 10^{5}$ time steps, the MSD has reached its equilibrium value for the curved interface (Fig. 4d), which it does not do until $t=2 \times 10^{6}$ time steps for the flat interface (Fig. 1d).

The explanation for the faster increase and equilibration of MSD is mainly due to that for a curved interface, receptor-ligand binding is possible only in a fraction of the total $3 \times 3 \mu \mathrm{m}$ domain, in contrast to a flat interface, where receptor-ligand binding is equally likely throughout the domain. The probability of dissociation $p_{\text {off }}$ is a minimum only at the center, and increases rapidly away from it. For example, for $P_{\mathrm{A}}=10^{2}, p_{\text {off }}=0.01$ everywhere for a flat interface, but for a curved interface this holds only at the very center of the domain, and $p_{\text {off }}$ increases rapidly away from the center, reaching a value of 1.0 before the domain boundary is reached. ${ }^{41}$ If a receptor-ligand complex wanders near the edge of the zone where binding is possible, it will dissociate with probability $p_{\text {off }} \gg 0.01$. The area-averaged value of $p_{\text {off }}$ is thus much higher for a curved interface, and thus the frequency of receptor-ligand complex dissociation is considerably higher for a curved interface. Compared to a flat interface, ligand molecules spend a greater proportion of time in the $p_{\text {diff( } \mathrm{F})}=1$ regime, and MSD increases faster.

Another significant difference between the results of Figs. 1 and 4 is the complete absence in Fig. 4 of the transient sub-diffusive behavior due to receptor-ligand binding. Purely diffusive behavior across all affinity values is observed at $t=10^{3}$ time steps (Fig. 4a), while sub-diffusive behavior across all affinity values is observed at $t=10^{4}$ time steps (Fig. 4b). The absence of transient sub-diffusive behavior due to receptor-ligand binding is due to the fact that kinetic equilibrium is reached very slowly, at around $t=2 \times 10^{5}$ time steps, as seen in Fig. 3b. This time scale is roughly the same as the time scale for equilibration of the MSD itself. Since kinetic equilibrium is reached at around the same time as dynamic equilibrium (MSD), the transition from the $p_{\text {diff(F) }}=1$ to the $p_{\text {diff(C) }}=0.1$ regime occurs very gradually, and any transient sub-diffusive behavior due to receptor-ligand binding is imperceptible. Furthermore, by $t=10^{4}$ time steps sub-diffusive behavior due to the finite-domain size and surface curvature has already set in, thereby masking any subsequent subdiffusive behavior due to receptor-ligand binding. 


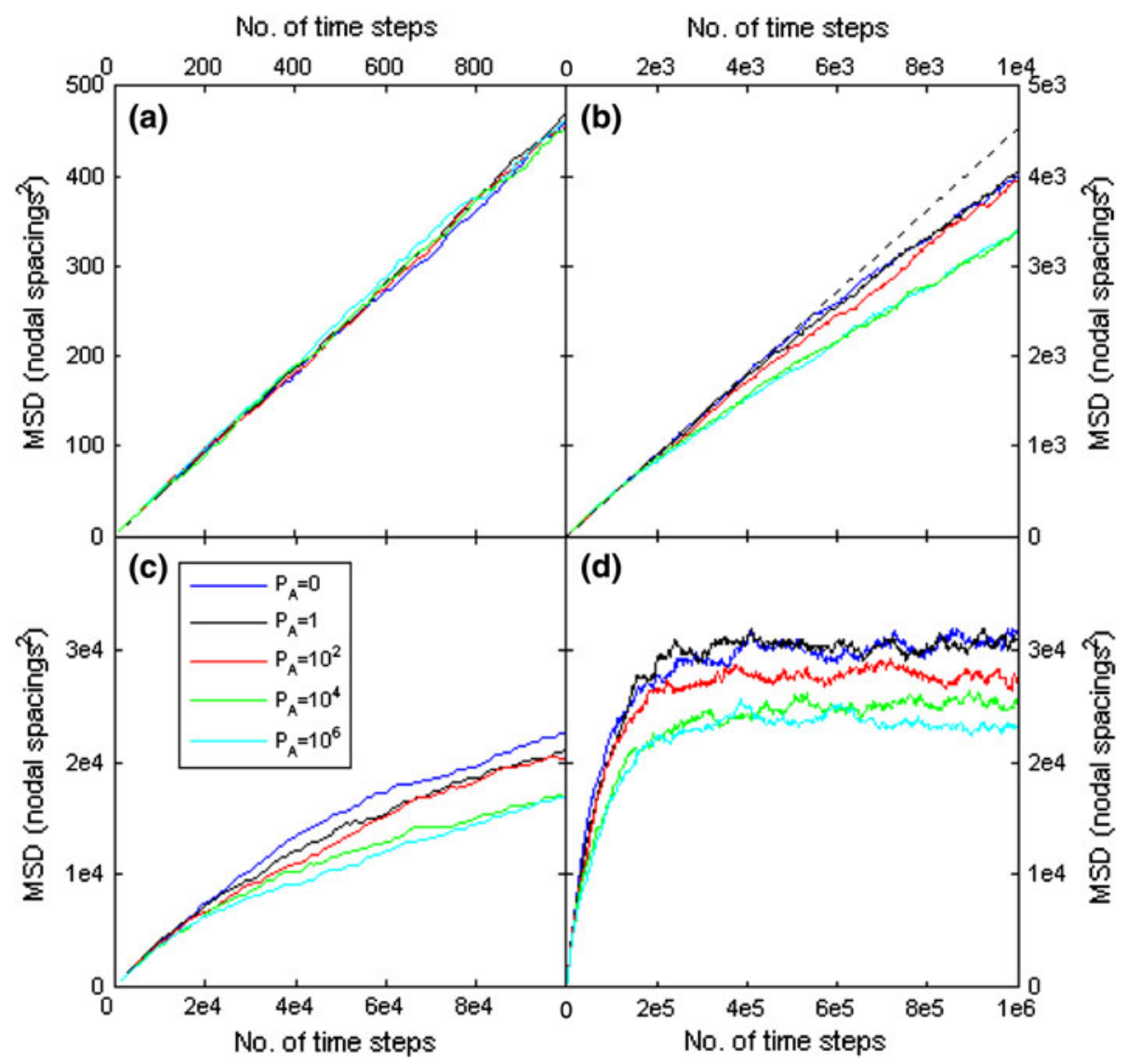

FIGURE 4. MSD as a function of time for unbiased diffusion and a curved interface. The simulation is run for $T=10^{6}$ time steps. Snapshots are taken after $t=10^{3}$ time steps (a), $t=10^{4}$ time steps (b), $t=10^{5}$ time steps (c), and $t=10^{6}$ time steps (d). Affinity takes on values of $P_{\mathrm{A}}=1,10^{2}, 10^{4}, 10^{6}$, and also includes a control with $P_{\mathrm{A}}=0$. The remaining parameters are given in Tables 1 and 2.

Perhaps the most noticeable difference between Figs. 1 and 4, however, is that as affinity increases, the equilibrium MSD value decreases (Fig. 4d). This is in marked contrast to the flat interface, where even though MSD increased more slowly with time as affinity increased, the equilibrium MSD value was the same for all affinity values (Fig. 1d). The decrease in the equilibrium value of MSD with affinity for a curved interface is due to the fact that $p_{\operatorname{diff}(\mathrm{C})}=0.1$ for receptor-ligand complexes but $p_{\operatorname{diff}(\mathrm{F})}=1$ for free ligands. For a curved interface, ligands that wander in the part of the domain where receptor-ligand binding is possible and bind to a receptor will experience a decrease in mobility by an order of magnitude and may thus remain near the center for a long time (trapping), thereby not reaching the maximum MSD possible (set by the domain size). As affinity increases, the region where receptor-ligand binding is possible becomes larger, ${ }^{41}$ and thus an increasing number of molecules remain near the center, and the molecule-averaged equilibrium MSD value is lower. Such trapping at the center of the domain is wholly absent in a flat contact interface, as $p_{\text {on }}$ and $p_{\text {off }}$ are constants throughout the domain. The decrease in equilibrium MSD with affinity is analogous to a decreasing diffusion exponent $(\alpha)$, indicating that interface curvature induces stronger sub-diffusive motion with increasing receptor-ligand binding affinity. Such affinity-dependent sub-diffusive motion is particularly important for studies on B cells, where the affinity of $\mathrm{B}$ cell receptors for antigens varies over five orders of magnitudes $\left(K_{\mathrm{A}}=10^{6}-10^{10} \mathrm{M}^{-1}\right)$ and contact between B cells and APCs initially takes place on protrusions that exhibit surface curvature. ${ }^{5,13}$ Given that the diffusion exponent decreases with affinity, in situations where kinetic parameters are not known it would theoretically be possible to extract such parameters from experimentally obtained log-log plots of MSD, provided a calibration between the diffusion exponent and kinetic parameters can be established. 


\section{Biased Diffusion}

\section{Single Surface, No Reaction}

In order to separate the effect of biased diffusion on MSD from the effect of receptor-ligand binding and interface curvature, we first perform simulations with biased diffusion on a single surface, without receptorligand binding. The evolution of MSD with time is shown in Fig. 5.

The difference between biased and unbiased diffusion is immediately apparent. At $t=10^{3}$ time steps (Fig. 5a), MSD for biased diffusion increases superdiffusively $(\alpha>1)$ and much more rapidly compared to unbiased diffusion, where MSD increases linearly with time. By $t=10^{4}$ time steps (Fig. 5b), the MSD for biased diffusion increases in a sub-diffusive manner, and in particular for the high bias cases of $\eta=1.5$ and 2 , it is approaching its equilibrium value. This is because at high bias, most molecules have already reached the center of the compartment by this time, and there is nowhere for them to diffuse to. Even if a molecule diffuses away from the center, it will quickly move back toward the center under the influence of bias. For unbiased diffusion, the MSD also increases sub-diffusively, albeit in a weaker manner and due exclusively to finite compartment size. We also note that MSD reaches its equilibrium value an order of magnitude faster when diffusion is biased (compare Fig. 5c, d), and that the equilibrium MSD value for biased diffusion is about half that for unbiased diffusion. This is due to the fact that molecules cluster at the center of the domain when diffusion is biased, causing them to "see" a smaller domain, similar to the "trapping" effect seen for unbiased diffusion on a curved contact interface discussed in the preceding section.

\section{Flat Contact Interface}

In Fig. 6, we show MSD as a function of time for a flat interface and weakly biased diffusion of receptorligand complexes $(\eta=1.1)$. Prior to $t=10^{4}$ time steps (Fig. 6a), the results are very similar to unbiased diffusion (Fig. 1a). At late times sub-diffusive behavior is

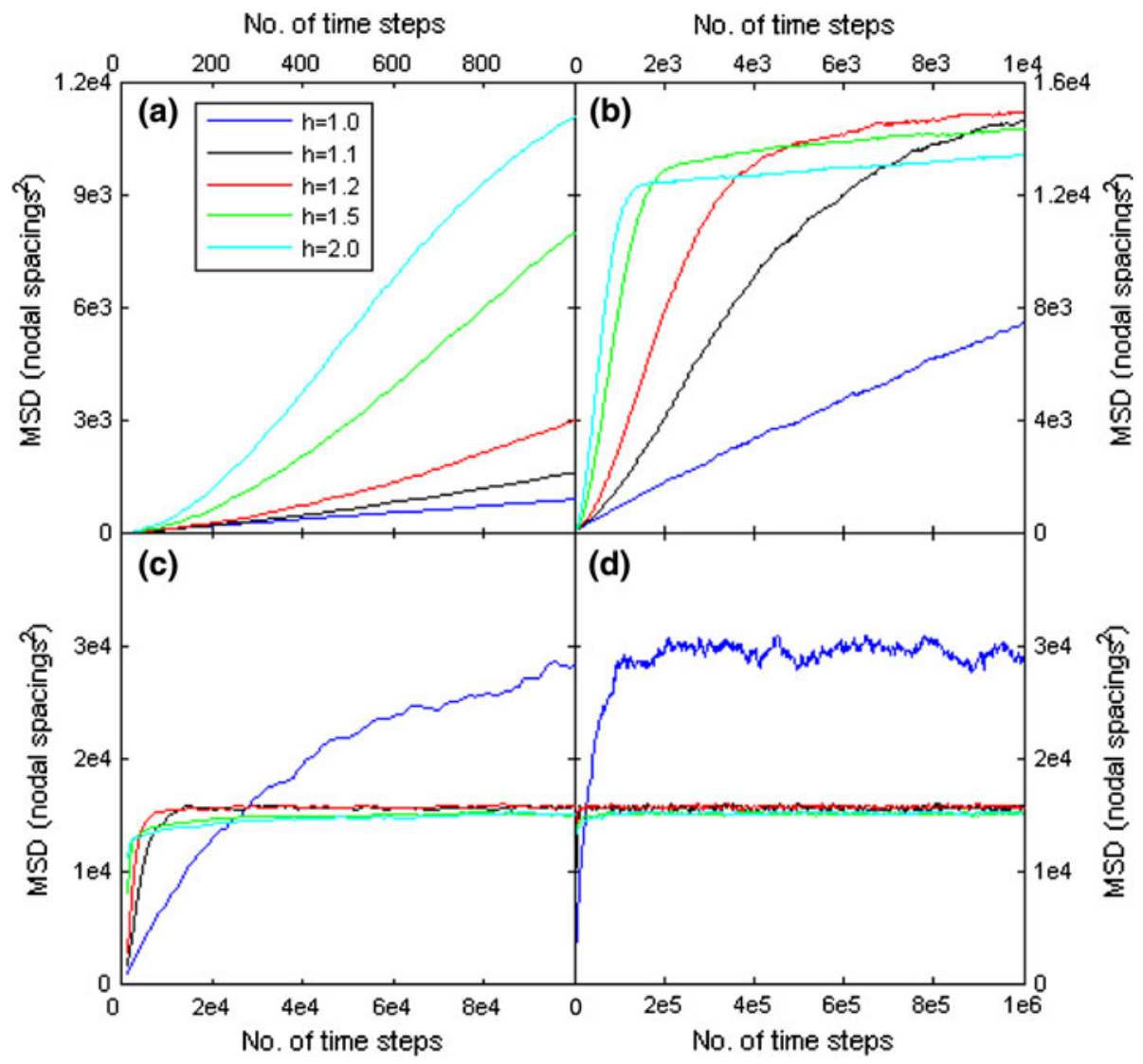

FIGURE 5. MSD as a function of time for biased diffusion on a single surface, without reaction. The simulation is run for $T=10^{6}$ time steps. Snapshots are taken after $t=10^{3}$ time steps (a), $t=10^{4}$ time steps (b), $t=10^{5}$ time steps (c), and $t=10^{6}$ time steps (d). In this figure there is no receptor-ligand binding, however, cytoskeletally mediated transport of molecules toward the center is simulated by means of biasing diffusion toward the center of the domain. The strength of the bias in diffusion ranges from $\eta=1.1$, $1.2,1.5,2.0$, with $\eta=1.0$ (no bias) included as a control. 


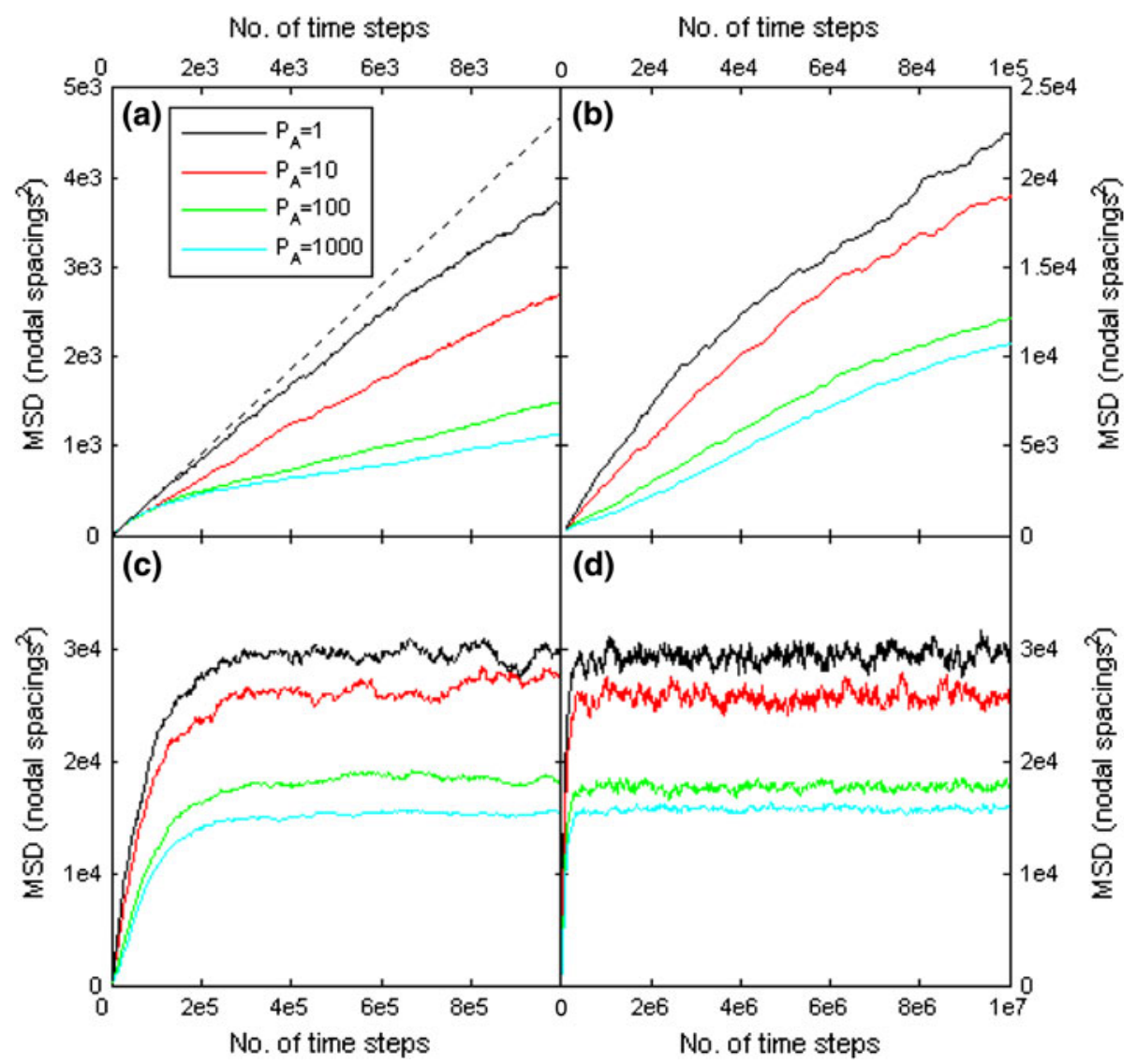

FIGURE 6. MSD as a function of time for weakly biased diffusion $(\eta=1.1)$ on a flat interface, with reaction. The simulation is run for $T=10^{7}$ time steps. Snapshots are taken after $t=10^{4}$ time steps (a), $t=10^{5}$ time steps (b), $t=10^{6}$ time steps (c), and $t=10^{7}$ time steps (d). Affinity takes on values of $P_{\mathrm{A}}=1,10,100,1000$, with $P_{\mathrm{A}}=1$ as a control since receptor-ligand binding is negligible at this affinity value and biased diffusion is undefined for $P_{\mathrm{A}}=0$.

apparent for all affinity values. The transient, early time sub-diffusive motion due to receptor ligand binding seen in Fig. 2 is also observable in Fig. 6a for $P_{\mathrm{A}}=10,100$, 1000. In a major difference from unbiased diffusion (Fig. 1), super-diffusive behavior is observed after $t=10^{4}$ time steps for high affinity $\left(P_{\mathrm{A}}=100,1000\right)$. Equilibrium is reached around $t=3 \times 10^{5}$ time steps (Fig. 6c), roughly the same as in Fig. 1. In a significant difference from Fig. 1, the time to equilibrium is the same for all affinity values, and the equilibrium MSD value decreases with increasing affinity.

At the lowest affinity value $\left(P_{\mathrm{A}}=1\right)$, too few receptor-ligand complexes form for the effect of biased diffusion to be noticeable. Rather, sub-diffusive motion is due exclusively to finite-domain size and the MSD curves are identical to those for unbiased diffusion (Fig. 1). The case of $P_{\mathrm{A}}=1$ can thus be thought of as a control (the case of $P_{\mathrm{A}}=0$ is not really suitable for a control, since biased diffusion only affects receptor-ligand complexes, thus for $P_{\mathrm{A}}=0$ biased diffusion is undefined).

The evolution of MSD as a function of time for strongly biased diffusion $(\eta=2.0)$ is shown in Fig. 7.
Strongly super-diffusive motion is observed from the start for high affinity $\left(P_{\mathrm{A}}=100,1000\right)$. Sub-diffusive motion due to receptor-ligand binding is absent, as the effect of strong bias completely negates it. Equilibrium is approached rapidly, however, there is a sharp transition to very slow sub-diffusive motion $t=2 \times 10^{4}$ time steps (Fig. 7b), and true equilibrium for high affinity is not reached till $t=2 \times 10^{6}$ time steps (Fig. 7d). As expected, the results for $P_{\mathrm{A}}=1$ are identical to those for weak bias (Fig. 6) and no bias (Fig. 1), since very few receptor-ligand complexes form at this affinity value.

At equilibrium, the MSD value for $P_{\mathrm{A}}=10,100$, and 1000 is about half that for $P_{\mathrm{A}}=1$, the reason being that when significant receptor-ligand binding occurs, most ligands cluster at the center of the contact zone as a result of strong bias and thus "see" a smaller domain. All sub-diffusive motion for these affinity values is due to bias toward the center, rather than finite-domain size. In contrast to the case of weak bias in Fig. 6, the equilibrium value does not decrease gradually with increasing affinity. With the exception of the control case of $P_{\mathrm{A}}=1$, for which no significant 
binding occurs, it is difficult to distinguish between $P_{\mathrm{A}}=10,100$, and 1000 at equilibrium.

\section{Curved Contact Interface}

Plots of MSD as a function of time for a curved interface and weakly biased diffusion of receptorligand complexes $(\eta=1.1)$ are shown in Fig. 8. The results are broadly similar to those for unbiased diffusion (Fig. 4). Purely diffusive motion at $t=10^{3}$ time steps (Fig. 8a) gives way to sub-diffusive motion by $t=10^{4}$ time steps (Fig. 8b). The only significant difference with respect to unbiased diffusion is that the decrease in equilibrium MSD with increasing affinity is more prominent here. This is because the effects of curvature and biased diffusion compound each other. As we saw in Fig. 4, curvature is by itself sufficient to induce clustering of receptor-ligand complexes at the center of the domain, causing ligand molecules to "see" a smaller domain. Biasing the diffusion of receptor-ligand complexes toward the center of the contact zone accelerates this process, resulting in a stronger decrease in equilibrium MSD value with increasing affinity.
Plots of MSD for strongly biased diffusion $(\eta=2.0)$ are shown in Fig. 9. Up until $t=10^{5}$ time steps (Fig. 9c), the plots in Fig. 9 are overall quite similar to those for unbiased diffusion (Fig. 4) and weakly biased diffusion (Fig. 8). Beginning at $t=2 \times 10^{5}$ time steps, however, for high affinity $\left(P_{\mathrm{A}}=10^{4}, 10^{6}\right)$ we observe a decrease in MSD immediately prior to the attainment of equilibrium at $t=4 \times 10^{5}$ time steps (Fig. 9d). The explanation for this phenomenon is as follows. For a curved interface, receptor-ligand complexes can only form within a fraction of the overall domain, where $p_{\text {on }}$ is high enough and $p_{\text {off }}$ sufficiently low. ${ }^{41}$ A receptorligand complex that forms at the edge of the zone where binding is possible will likely diffuse under the influence of bias to the center of the contact zone. It may, however, overshoot the center and once again enter the zone where $p_{\text {off }}$ is large on the opposite side. If the complex wanders back toward the center, its MSD will decrease, as it is moving in this case closer to its original position. If on the other hand it dissociates (with high probability if it diffuses in the zone where $p_{\text {off }}$ is high), the ligand molecule may, after some time, bind another receptor or re-bind its original partner and once again be transported toward the center, in

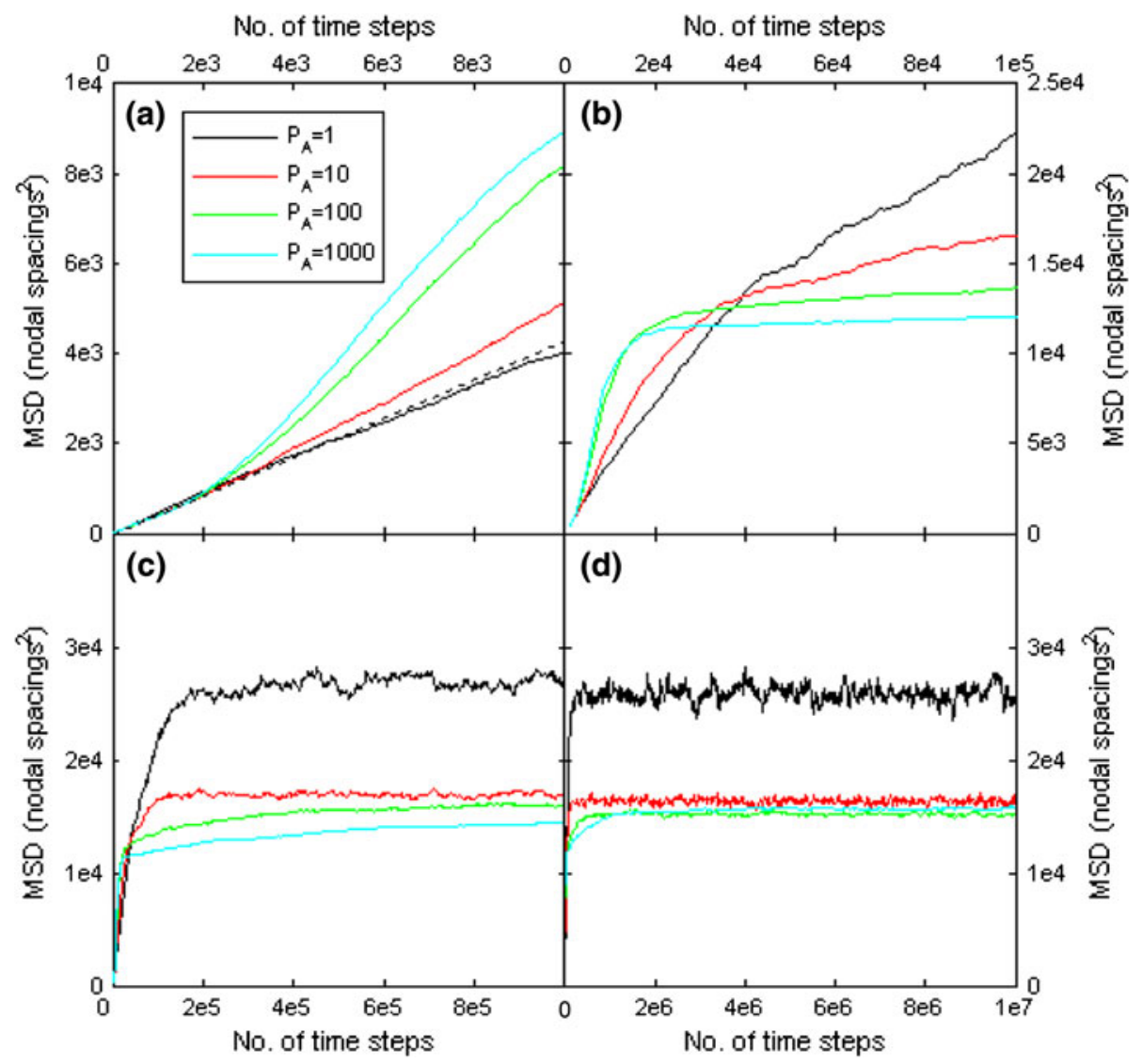

FIGURE 7. MSD as a function of time for strongly biased diffusion $(\eta=2.0)$ on a flat interface, with reaction. The simulation is run for $T=10^{7}$ time steps. Snapshots are taken after $t=10^{4}$ time steps (a), $t=10^{5}$ time steps (b), $t=10^{6}$ time steps (c), and $t=10^{7}$ time steps (d). 


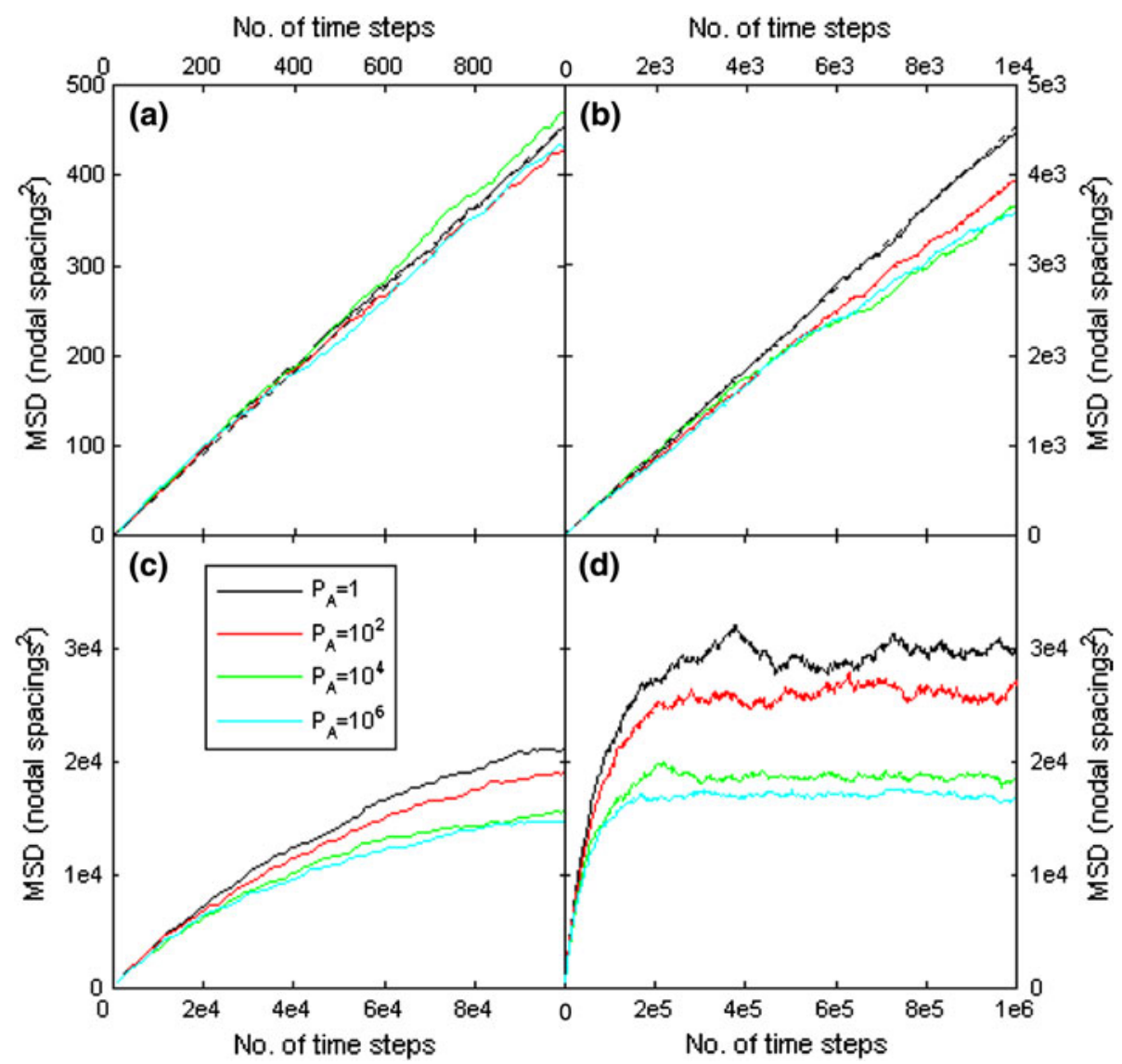

FIGURE 8. MSD as a function of time for weakly biased diffusion $(\eta=1.1)$ on a curved interface, with reaction. The simulation is run for $T=10^{6}$ time steps. Snapshots are taken after $t=10^{3}$ time steps (a), $t=10^{4}$ time steps (b), $t=10^{5}$ time steps (c), and $t=10^{6}$ time steps (d).

which case its MSD will also decrease before settling to its equilibrium value. This effect can only occur with a curved contact interface, because for a flat contact interface $p_{\text {off }}$ is uniformly low (at high affinity) throughout the domain, hence the likelihood of dissociation is uniformly low throughout the domain. Bias is necessary for this phenomenon, because at high affinity for a curved interface, in the absence of bias most receptor-ligand complexes will form at the edge of the zone where binding is possible and stay there, forming a ring-like structure. ${ }^{41}$ This effect is also only observed at high affinity, because at low affinity most molecules will stay free, and hence will not be transported to the center.

\section{Quantification of Clustering}

Given that clustering of lymphocyte receptors and antigens on the surface of an APC is a crucial step in the lymphocyte activation pathway, $1,5,10,13,14,16,19,25,36,38,46$ we investigate how diffusion on a cell membrane impacts clustering. As a metric of clustering, we use the mean distance of ligands from the center of the contact zone. In Fig. 10, we tabulate mean distance from the center for a flat interface (Fig. 10a) and a curved interface (Fig. 10b) for unbiased $(\eta=1.0)$, weakly biased $(\eta=1.1)$, and strongly biased diffusion $(\eta=2.0)$. For unbiased diffusion across a flat interface, the mean distance from the center does not change with affinity, fluctuating around a value of 120 nodal spacings. This is the value corresponding to uniform distribution (a circle with this radius has half the area of a $300 \times 300$ square), indicating the complete absence of clustering in this case. When diffusion is biased toward the center, the mean distance of ligands from the center decreases with affinity, more steeply so with stronger bias. For weak bias $(\eta=1.1)$, we observe a steep decrease in mean distance from the center when affinity increases from $P_{\mathrm{A}}=10$ to $P_{\mathrm{A}}=100$. For strong bias $(\eta=2.0)$, a similar steep decrease is observed going from $P_{\mathrm{A}}=1$ to $P_{\mathrm{A}}=10$. The mean distance from the center following the steep decrease is $\sim 40$ or less nodal spacings, indicating strong clustering (in previous work, we showed that significant clustering occurs when the mean distance of the center is around half value for uniform distribution $\left.{ }^{40}\right)$. 


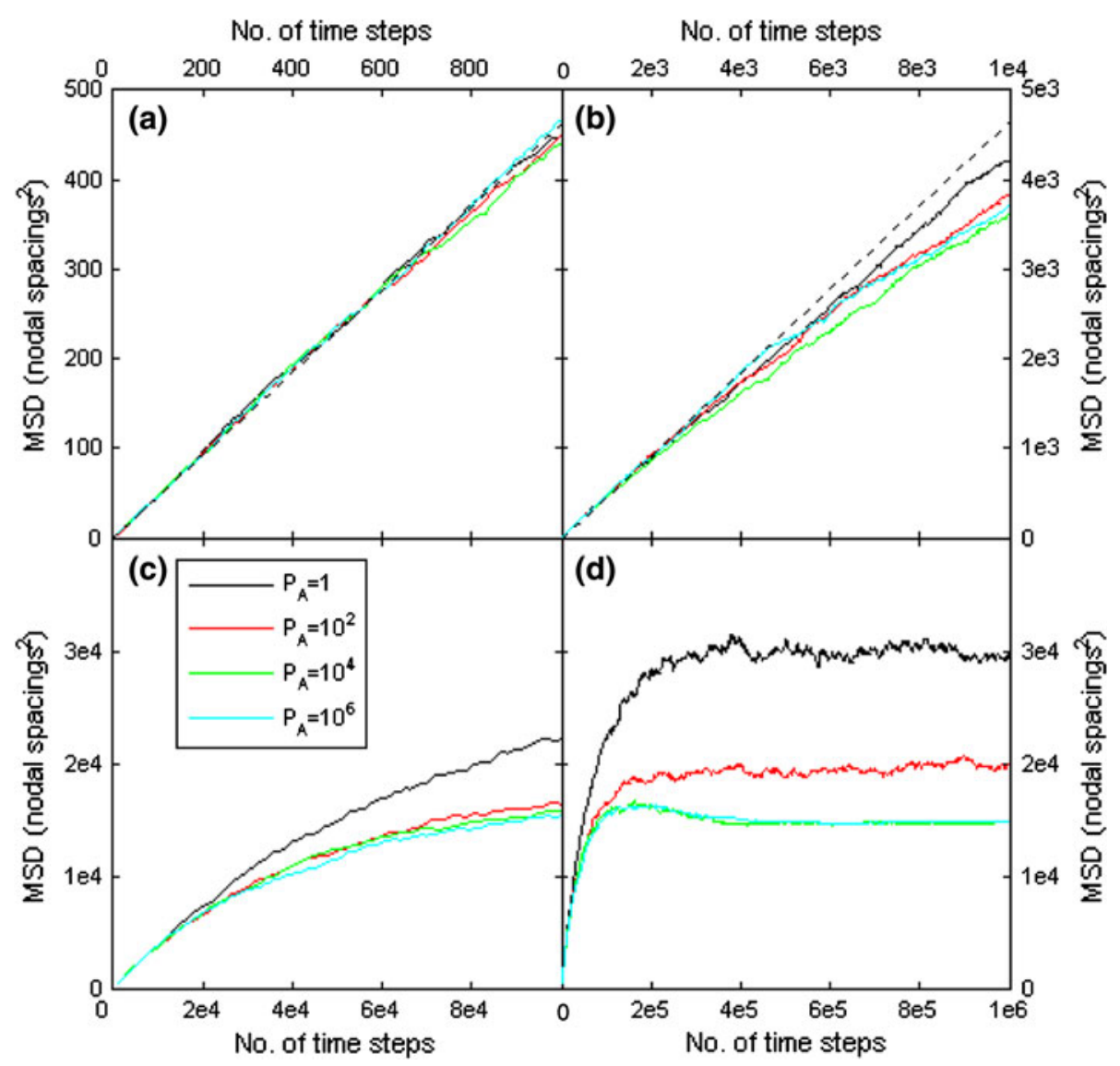

FIGURE 9. MSD as a function of time for strongly biased diffusion $(\eta=2.0)$ on a curved interface, with reaction. The simulation is run for $T=10^{6}$ time steps. Snapshots are taken after $t=10^{3}$ time steps (a), $t=10^{4}$ time steps (b), $t=10^{5}$ time steps (c), and $t=10^{6}$ time steps (d).

For a curved interface, the mean distance from the center decreases with affinity even for unbiased diffusion, albeit weakly. This reflects the trapping of receptor-ligand complexes in the zone where binding is possible observed in the MSD curves of Fig. 4. The results for biased diffusion are strikingly similar to those for biased diffusion for a flat interface: a steep decrease in the mean distance from the center when affinity increases from $P_{\mathrm{A}}=10^{2}$ to $P_{\mathrm{A}}=10^{4}$ for weak bias, and when affinity increases from $P_{\mathrm{A}}=1$ to $P_{\mathrm{A}}=10^{2}$ for strong bias. Taken together, these results indicate that directed transport toward the center of the contact interface appears necessary for significant clustering to occur, no matter the geometry of the interface.

The reason clustering is not very strong for a curved interface for unbiased diffusion (Fig. 9b) is that receptor-ligand complexes tend to form near the edges of the zone where binding is possible, rather than at the very center. This is because most receptor-ligand complexes form as free receptors and ligands diffuse from outside the zone of binding, where most of them are initially located, and encounter a binding partner.
As they diffuse slower once they bind, many receptorligand complexes remain near where they formed, in the outer regions of the zone of binding, rather than diffuse to the center. Thus, clustering for unbiased diffusion on a curved interface is weaker than for biased diffusion, as in the latter case the receptorligand complexes cluster tightly at the very center of the contact region due to the influence of bias.

\section{DISCUSSION}

In this study, we investigated diffusion of receptors and ligands that bind to each other across two opposing surfaces, as is the case during cell-cell or cell-bilayer interactions. Cell-cell interactions are key to recognition of foreign pathogens by $\mathrm{T}$ and $\mathrm{B}$ lymphocytes. Immune receptors such as TCR or BCR have evolved to sense antigen affinity. Our results indicate diffusion of receptors that bind to ligands on an opposing surface is very much affinity dependent and therefore influences the ability of cells to sense antigen affinity in a manner similar to positive feedback. 


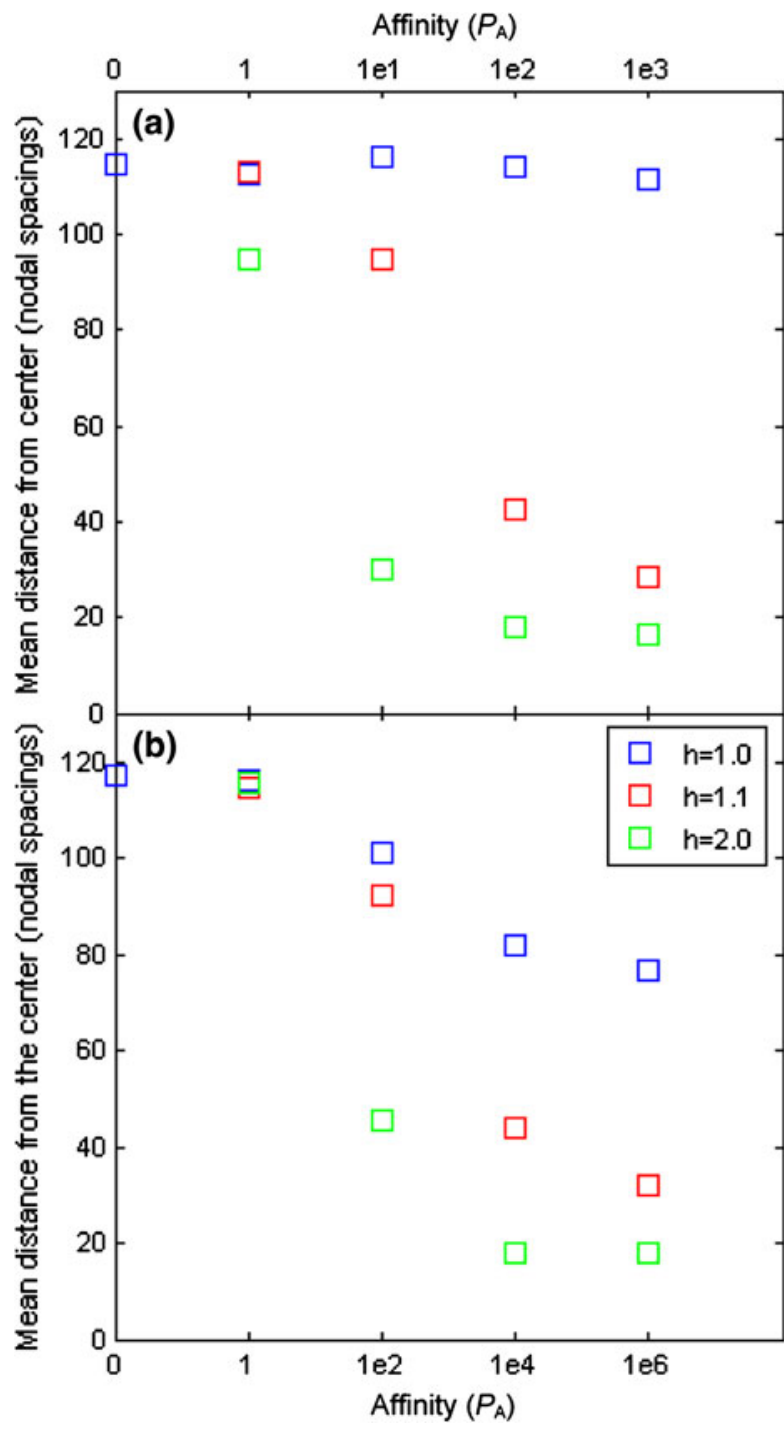

FIGURE 10. Mean distance from the center of ligand molecules for a flat interface (Fig. 9a) and a curved interface (Fig. 9b). Mean distance from the center is used a measure of clustering.

Our results show the MSD of tracked ligand molecules, as can be obtained from single particle-tracking experiments, is a powerful tool for probing the nature of diffusion across a cell-cell interface. Plots of MSD can reveal information about the shape of the interface. For a completely flat interface, the MSD reaches the same equilibrium value regardless of receptorligand binding affinity. By contrast, should either or both surfaces exhibit curvature, our results show a decrease in the equilibrium value of the MSD as receptor-ligand binding affinity increases. Higher surface curvature makes the affinity-dependent decrease in the equilibrium value of MSD more pronounced.

The evolution of MSD as a function of time can also be used to test for directed transport of receptors toward a specific point on the cell surface, as is known to happen during antigen recognition by lymphocytes. For a flat interface, super-diffusive motion is detected at early times. By contrast, super-diffusive motion is never observed for a curved interface, even with strong bias. For a curved interface and high affinity binding, an unmistakable decrease in MSD with time is observed prior to equilibration for high-affinity values. Both effects can be used to detect the presence of directed transport, as well as to determine whether the interface is flat or curved. The decrease in the diffusion exponent, $\alpha$, with affinity that can be observed in our simulations could also lead to the development of a theoretical method for estimating kinetic parameters (when they are not known) from $\log -\log$ plots of MSD.

Experimental and theoretical studies show that membrane heterogeneity can affect transport of receptor proteins diffusing on a cell surface. In this study, we have shown that sub-diffusive motion can arise from effects associated with receptor-ligand binding across two opposing surface. For a flat interface, receptor-ligand binding causes transient sub-diffusive motion at early times, provided receptor-ligand complexes diffuse slower than free molecules. This effect disappears once kinetic equilibrium is reached, in which case motion is purely diffusive albeit with a lower diffusion coefficient. Such an effect is entirely absent when the interface between the two surfaces exhibits curvature. However, in this case, interface curvature induces sub-diffusive behavior, the result of which is the decrease in the equilibrium value of MSD with increasing receptor-ligand binding affinity.

Also of note is that directed transport induces a decrease in the equilibrium value of MSD as receptorligand binding affinity increases, similar to what is seen for unbiased diffusion with a curved interface. This holds true even for a flat interface, which was not the case with unbiased diffusion. For weak bias, the decrease in equilibrium MSD with affinity is gradual, however, for strong bias values, it becomes difficult to distinguish among affinity values for which significant receptor-ligand binding occurs. Our results also indicate that some form of directed transport is necessary for significant clustering of receptors and ligands to occur.

\section{ACKNOWLEDGMENTS}

S.R. and P.T. are supported by NIH grant AI074022. The authors thank Dr. Scott Simon for carefully reading the manuscript. 


\section{OPEN ACCESS}

This article is distributed under the terms of the Creative Commons Attribution Noncommercial License which permits any noncommercial use, distribution, and reproduction in any medium, provided the original author(s) and source are credited.

\section{REFERENCES}

${ }^{1}$ Batista, F. D., D. Iber, and M. S. Neuberger. B cells acquire antigen from target cells after synapse formation. Nature 411:489-494, 2001.

${ }^{2}$ Bell, G. I. Cell-cell adhesion in the immune system. Immunol. Today 4:237-240, 1983.

${ }^{3}$ Burrage, K., J. Hancock, A. Leier, and D. V. Nicolau. Modeling and simulation techniques for membrane biology. Brief. Bioinform. 8:234-244, 2007.

${ }^{4}$ Burroughs, N. J., and C. Wülfing. Differential segregation in a cell-cell contact interface: the dynamics of the immunological synapse. Biophys. J. 83:1784-1796, 2002.

${ }^{5}$ Carrasco, Y. R., S. J. Fleire, T. Cameron, M. L. Dustin, and F. D. Batista. LFA-1/ICAM-1 interaction lowers the threshold of B cell activation by facilitating B cell adhesion and synapse formation. Immunity 20:589-599, 2004.

${ }^{6}$ Cherry, R. J., P. R. Smith, I. E. G. Morrison, and N. Fernandez. Mobility of cell surface receptors: a re-evaluation. FEBS Lett. 430:88-91, 1998.

${ }^{7}$ Das, R., C. Cairo, and D. Coombs. A hidden Markov model for single particle tracks quantifies dynamic interactions between LFA-1 and the actin cytoskeleton. PLoS Comput. Biol. 5:1-16, 2010.

${ }^{8}$ Dembo, M., T. C. Torney, K. Saxman, and D. Hammer. The reaction-limited kinetics of membrane-to-surface adhesion and detachment. Proc. R. Soc. Lond. B 234:5583, 1998.

${ }^{9}$ DeMond, A. L., K. D. Mossman, T. Starr, M. L. Dustin, and J. T. Groves. T cell receptor microcluster transport through molecular mazes reveals mechanism of translocation. Biophys. J. 94:3286-3292, 2008.

${ }^{10}$ Depoil, D., S. J. Fleire, B. L. Treanor, M. Weber, and N. E. Harwood. CD19 is essential to B cell activation by promoting $\mathrm{B}$ cell receptor-antigen micro-cluster formation in response to membrane-bound ligand. Nat. Immunol. 9:63-72, 2008.

${ }^{11}$ Dilger, J. P. Monte Carlo simulation of buffered diffusion into and out of a model synapse. Biophys. J. 98:959-967, 2010.

${ }^{12}$ Favier, B., N. J. Burroughs, L. Weddeburn, and S. Valitutti. $\mathrm{T}$ cell antigen receptor dynamics on the surface of living cells. Int. Immunol. 13:1525-1532, 2002.

${ }^{13}$ Fleire, S. J., J. P. Goldman, Y. R. Carrasco, M. Weber, D. Bray, et al. B cell ligand discrimination through a spreading and contracting response. Science 312:738-741, 2006.

${ }^{14}$ Grakoui, A., S. K. Bromley, C. Sumen, M. M. Davis, A. S. Shaw, et al. The immunological synapse: a molecular machine controlling T cell activation. Science 285:221-227, 1999.

${ }^{15}$ Iber, D. Formation of the B-cell synapse: retention or recruitment? Cell Mol. Life Sci. 62:206-213, 2005.

${ }^{16}$ Kaizuka, Y., A. Douglass, R. Varma, M. L. Dustin, and R. D. Vale. Mechanisms for segregating $T$ cell receptor and adhesion molecules during immunological synapse formation in Jurkat T cells. Proc. Natl. Acad. Sci. USA 104: 20296-20301, 2007.

${ }^{17}$ Kim, J. S., and A. Yethiraj. Crowding effects on association reactions at membranes. Biophys. J. 98:951-958, 2010.

${ }^{18}$ Krobath, H., G. J. Schütz, R. Lipowsky, and T. R. Weikl. Lateral diffusion of receptor-ligand bonds in membrane adhesion zones: effect of thermal membrane roughness. Eur. Phys. Lett. 78:38003, 2007.

${ }^{19}$ Krummel, M. F., M. D. Sjaastad, C. Wülfing, and M. M. Davis. Differential clustering of CD4 and CD3 $\zeta$ during T cell recognition. Science 289:1349-1352, 2000.

${ }^{20}$ Kusumi, A., H. Ike, C. Nakada, M. Kotono, and T. Fujiwara. Single-molecule tracking of membrane molecules: plasma membrane compartmentalization and dynamic assembly of raft-philic signaling molecules. Semin. Immunol. 17:3-21, 2005.

${ }^{21}$ Kusumi, A., C. Nakada, K. Ritchie, K. Murase, K. Suzuki, et al. Paradigm shift of the plasma membrane concept from the two-dimensional continuum fluid to the partitioned fluid: high-speed single-molecule tracking of membrane molecules. Annu. Rev. Biophys. Biomol. Struct. 34:351-378, 2005.

${ }^{22}$ Kusumui, A., and Y. Suzuki. Toward understanding the dynamics of membrane-raft-based molecular interactions. Biochim. Biophys. Acta 1746:234-251, 2005.

${ }^{23}$ Lauffenburger, D. A., and J. J. Linderman. Models for Binding, Trafficking and Signaling. Oxford, UK: Oxford University Press, 365 pp, 1993.

${ }^{24}$ Liu, W., T. Meckel, P. Tolar, H. W. Sohn, and S. K. Pierce. Antigen affinity discrimination is an intrinsic function of the B cell receptor. J. Exp. Med. 207:1095-1111, 2010.

${ }^{25}$ Monks, C. R., B. A. Freiberg, H. Kupfer, N. Sciaky, and A. Kupfer. Three-dimensional segregation of supramolecular activation clusters in T cells. Nature 395:82-86, 1998.

${ }^{26}$ Nicolau, D. V., J. Hancock, and K. Burrage. Sources of anomalous diffusion on cell membranes: a Monte Carlo study. Biophys. J. 92:1975-1987, 2007.

${ }^{27}$ Niehaus, A. M. S., D. G. Vlachos, J. S. Edwards, P. Pletchac, and R. Tribe. Microscopic simulation of membrane molecule diffusion on corralled membrane surfaces. Biophys. J. 94:1551-1564, 2008

${ }^{28}$ Raychaudhuri, S., P. K. Tsourkas, and E. Willgohs. Computational modeling of receptor-ligand binding and cellular signaling processes. In: Handbook of Modern Biophysics, Volume I Fundamentals, edited by T. Jue. New York: Humana Press, 2009, pp. 41-61.

${ }^{29}$ Saxton, M. J. Single-particle tracking: models of directed transport. Biophys. J. 67:2110-2119, 1994.

${ }^{30}$ Saxton, M. J. Single-particle tracking: effects of corrals. Biophys. J. 69:389-398, 1995.

${ }^{31}$ Saxton, M. J. Anomalous diffusion due to binding: a Monte Carlo study. Biophys. J. 70:1250-1262, 1996.

${ }^{32}$ Saxton, M. J. Anomalous subdiffusion in fluorescence photobleaching recovery: a Monte Carlo study. Biophys. J. 81:2226-2240, 2001.

${ }^{33}$ Saxton, M. J. A biological interpretation of transient anomalous subdiffusion. I. Qualitative model. Biophys. J. 92:1178-1191, 2007.

${ }^{34}$ Saxton, M. J. A biological interpretation of transient anomalous subdiffusion. II. Reaction kinetics. Biophys. J. 94:760-771, 2008.

${ }^{35}$ Saxton, M. J., and K. Jacobson. Single-particle tracking: applications to membrane dynamics. Annu. Rev. Biophys. Biomol. 26:373-399, 1997.

${ }^{36}$ Sohn, H. W., P. Tolar, and S. K. Pierce. Membrane heterogeneities in the formation of $\mathrm{B}$ cell receptor-Lyn kinase 
microclusters and the immune synapse. J. Cell Biol. 182:367-379, 2008.

${ }^{37}$ Sung, B. J., and A. Yethiraj. Computer simulations of protein diffusion in compartmentalized cell membranes. Biophys. J. 97:472-479, 2009.

${ }^{38}$ Tolar, P., H. W. Sohn, and S. K. Pierce. Viewing the antigen induced initiation of $\mathrm{B}$ cell activation in living cells. Immunol. Rev. 221:64-76, 2008.

${ }^{39}$ Tolentino, T. P., J. Wu, V. I. Zarnitsyna, Y. Fang, M. L. Dustin, et al. Measuring diffusion and binding kinetics by contact area FRAP. Biophys. J. 95:920-930, 2008.

${ }^{40}$ Tsourkas, P. K., and S. Raychaudhuri. Modeling of B cell synapse formation by Monte Carlo simulation shows that directed transport of receptor molecules is a potential formation mechanism. Cell. Mol. Bioeng. 3:256-268, 2010.

${ }^{41}$ Tsourkas, P. K., N. Baumgarth, S. I. Simon, and S. Raychaudhuri. Mechanisms of B cell synapse formation predicted by Monte Carlo simulation. Biophys. J. 92:41964208, 2007.
${ }^{42}$ Tsourkas, P. K., M. L. Longo, and S. Raychaudhuri. Monte Carlo study of single molecule diffusion can elucidate the mechanism of B cell synapse formation. Biophys. $J$. 95:1118-1125, 2008.

${ }^{43}$ Wang, B., S. M. Anthony, S. C. Bae, and S. Granick. Anomalous yet Brownian. Proc. Natl. Acad. Sci. USA 106:15160-15164, 2009.

${ }^{44}$ Wieser, S., M. Axmann, and G. J. Schütz. Versatile analysis of single-molecule tracking data by comprehensive testing against Monte Carlo simulations. Biophys. J. 95:59886001, 2008.

${ }^{45}$ Wülfing, C., and M. M. Davis. A receptor/cytoskeletal movement triggered by co-stimulation during $\mathrm{T}$ cell activation. Science 282:2266-2269, 1998.

${ }^{46}$ Wülfing, C., M. D. Sjaastad, and M. M. Davis. Visualizing the dynamics of $\mathrm{T}$ cell activation: intracellular adhesion molecule 1 migrates rapidly to the $\mathrm{T}$ cell/B cell interface and acts to sustain calcium levels. Proc. Natl. Acad. Sci. USA 95:6302-6307, 1998. 